als Experten die Harvard-Koryphäen aufgeboten werden. Da ist ein Markt entstanden, der nicht mit armseligen Beratungshonoraren auskommt: $\$ 745$ sind zu berappen, wenn die Zweitmeinung - online! - auch zum pathologischen Befund Stellung bezieht. Die Cleveland Clinic steht dem nicht nach, macht aber gleich darauf aufmerksam, dass die Kosten von Medicare nicht getragen werden und man sich außerstande sieht, die Honorare von Versicherungen zu akzeptieren. Eine ziemlich maliziöse Variante darf nicht unterschlagen werden: Die Institution verzichtet auf ein Honorar für die Zweitmeinung, gibt sich aber mit Spenden zufrieden, "etwa mit einer nicht mehr benötigten Lebensversicherung, Wohnung, Farm, Kunstobjekte, Aktien..." Die amerikanische Ärztekammer scheint in einem Dilemma: Sie möchte die online-Beratungen nicht blockieren, spricht sich aber unverhohlen für ein persönliches ärztliches Gespräch aus.

\section{Krankenkassen machen Angebote}

Und der deutsche Weg? Die AOK BadenWürttemberg engagiert sich, denn „sie lässt ihre Versicherten im Fall der Fälle nicht allein“ und vermittelt „Top-Ärzte“ „zur Zweitmeinung für die Bereiche Orthopädie, Urologie und Onkologie. Der Termin wird innerhalb von acht Tagen vermittelt." Die Techniker Krankenkasse bietet ein ZweitmeinungsTelefon an: „,kompetente Fachärzte rund um die Uhr." Auch die Online-Firma "Medführer" hat diese Lücke entdeckt und bietet Kompetenz an. Ein Argument: „Nicht zuletzt hilft ein Zweitbefund einem Patienten, den richtigen Facharzt für eine Diagnose oder Therapie zu finden." Die "Unabhängige Patientenberatung Deutschland“ mahnt, für die Zweitmeinung einen kassenzugelassenen Arzt auszuwählen, dann übernimmt die Kasse die Kosten (verbreitet in der Bild-Zeitung vom 26.9.2012). Eine Parallelwelt mit Zweitmeinungen lehnt Katus kategorisch ab. Der Qualitätsstandard in Deutschland sieht Leitlinien, Qualitätskontrollen und ein Konsiliarsystem vor. Das sollte reichen, diese Absicherungen müssten nur voll ausgeschöpft werden. Gestärkt werden sollte allerdings die kooperative Therapieplanung und kontrolliert werden sollten Fehlanreize wie Selbstzuweisung sowie auch die Expansion von Leistungen. Er fasste zusammen:

- Zweitmeinungen sind ein Eminenz-getriebener Ansatz, der sich auf keine evidenzbasierten Daten stützen kann und vom
"Schiedsrichter" erhebliche Verantwortung verlangt.

- Der Zugang zur Zweitmeinung ist ethisch problematisch und kann Ärzte in eine defensive Entscheidungshaltung drängen. Dass versteckte finanzielle Interessen dahinter stecken können, ist häufig mehr als wahrscheinlich. Katus setzt voraus, dass eine Zweitmeinung folgende Kriterien erfüllt:

- Komplette Beurteilung aller klinischen Variablen und Qualität der Befunde,

- Kenntnis der diagnostischen und therapeutischen Alternativen,

- Berücksichtigung der regionalen Versorgungsstruktur und persönlicher Komorbidität sowie der Lebenssituation.
- Nicht zuletzt wird vom Schiedsrichter vorausgesetzt , "blinded" und „unbiased" sein.

Die Kontroversen auf der DGK-Herbsttagung verlangen nach Fortsetzung, sie haben prinzipielle Fragen diskutiert, die mit einer "Talk-Show" nicht ausreichend gewürdigt sein können.

Bemerkenswert war das Engagement der Kontrahenten, sie wollten überzeugen und nicht nur an der Oberfläche der Probleme kratzen.

Dr. med. Jochen Aumiller II

II Quelle: DGK-Herbsttagung und Jahrestagung der Arbeitsgruppe Rhythmologie vom 11.-13. Oktober 2012 in Hamburg

\title{
Checklisten auch in der Kardiologie?
}

Standardisierte Behandlungsabläufe auf der Basis von Checklisten kann in kardiologischen Abteilungen die Dauer des Klinikaufenthalts bei Herzpatienten zum Teil deutlich verkürzen. Das zeigt eine Studie des Universitätsklinikums Schleswig-Holstein, Lübeck.

Das Lübecker Team entwickelte solche Checklisten für eine Reihe kardiologischer Diagnosen und verglich die durchschnittliche Aufenthaltsdauer von Patienten vor und nach Einführung des Systems. Bei allen beobachteten Erkrankungen waren die Klinikaufenthalte der Patienten nach der Einführung der Checklisten kürzer als vorher: Minus 4 Prozent beim akuten Koronarsyn- drom, minus 7,5 Prozent beim Vorhofflimmern, minus 6,1 Prozent bei der Herzinsuffizienz. Bei seltener auftretenden Diagnosen wie tiefen Venenthrombosen (minus 11 Prozent) oder Lungenarterienembolien (minus 28 Prozent) fiel die Reduktion noch deutlicher aus.

„Die Ergebnisse zeigen, dass mit dem vorgestellten checklistenbasierten System auch in der Inneren Medizin eine breite Implementierung von Behandlungspfaden möglich ist", fassen die Autoren zusammen. Gerade bei seltener vorkommenden Diagnosen erscheine eine Prozessoptimierung möglich.

(J.A.) II

\section{Online Depressions-Vorbeugung bei ICD}

Iـ Studien belegen, dass ICD-Patienten trotz des erfolgreichen Eingriffs häufig an Depressionen und Angst leiden. Dagegen ließen sich die Bremer Kardiologen etwas einfallen. Sie stellten ICD-Patienten für zwölf Wochen online das therapeutische Unterstützungsprogramm „Novego-Depressionshelfer" zur Verfügung. Das Programm bietet für ICD- und Schrittmacher-Träger individualisierte Inhalte, begleitet von psychologischen Gesprächen und Übungsfeedback. Zu den Inhalten gehören ärztlicher Rat von Kardiologen und Elektrophysiologen, Wissen über Herzerkrankungen, Ängste und Depression sowie Übungen und Alltagstipps.
ICD-Patienten hatten nach Absolvierung des Programms deutlich weniger Ängste und depressive Symptome. Weitere positive Effekte bei den Online-Patienten: Sie verändern stärker ihre Lebensweise, handeln aktiv gegen ihre Ängste und entwickeln eine hohe Akzeptanz des Defibrillators als lebensrettendes technisches Instrument. „Eine psychokardiologische Unterstützung ist auch in Internet-basierten therapeutischen Programmen möglich und kann eine effektive Unterstützung des Elektrophysiologen und Kardiologen bei der Nachbetreuung der Patienten bieten", schlussfolgern Muth-Seidel und Mitarbeiter.

(J. A.) । 